\title{
Comprehensive Analysis of MicroRNA and mRNA Expression in Normal and Tumorous Human Esophageal Squamous Cell Lines Using Microarray Datasets
}

\author{
Ichiro Akagi, ${ }^{1}$ Osamu Ishibashi, ${ }^{2}$ Takeshi Matsutani, ${ }^{3}$ \\ Nobutoshi Hagiwara, ${ }^{3}$ Akihisa Matsuda, ${ }^{4}$ Tsutomu Nomura, ${ }^{3}$ Hiroshi Makino, ${ }^{1}$ \\ Hiroshi Yoshida, ${ }^{1}$ Masao Miyashita, ${ }^{4}$ and Eiji Uchida ${ }^{3}$ \\ ${ }^{1}$ Department of Surgery, Nippon Medical School Tama Nagayama Hospital, Tokyo 206-8512, Japan \\ ${ }^{2}$ Laboratory of Biological Macromolecules, Graduate School of Life and Environmental Sciences, Osaka Prefecture University, Sakai \\ 599-8531, Japan \\ ${ }^{3}$ Department of Gastrointestinal and Hepato-Biliary-Pancreatic Surgery, Nippon Medical School, Tokyo 113-8602, Japan \\ ${ }^{4}$ Department of Surgery, Nippon Medical School Chiba Hokusou Hospital, Inzai, Chiba 270-1694, Japan
}

Correspondence should be addressed to Ichiro Akagi; ichiro@nms.ac.jp and Osamu Ishibashi; ishibashi@biochem.osakafu-u.ac.jp Received 5 October 2014; Accepted 13 November 2014; Published 15 December 2014

Academic Editor: Qinghua Cui

Copyright (c) 2014 Ichiro Akagi et al. This is an open access article distributed under the Creative Commons Attribution License, which permits unrestricted use, distribution, and reproduction in any medium, provided the original work is properly cited.

\begin{abstract}
Despite the undisputed importance of altered microRNA (miRNA) expression in various cancers, there is limited information on the clinicopathologic significance of cancer-related miRNAs in esophageal squamous cell carcinoma (ESCC). Previously, it was reported that the expression of several miRNAs was dysregulated in ESCC. However, the target genes of these miRNAs have not been identified. Furthermore, additional miRNAs in humans have been discovered recently, indicating that revised miRNA and gene expression profiling for ESCC are necessary. Here, we provide datasets from microarray analyses to identify miRNA and mRNA expression comprehensively in Het-1A, a normal human esophageal squamous cell line, and three human ESCC cell lines.
\end{abstract}

\section{Introduction}

Esophageal cancer (EC) is the sixth most common cancer in the world that predominantly affects men and has a poor prognosis [1]. EC is classified mainly into two typessquamous cell carcinoma and adenocarcinoma-which begin as flat cells lining the esophagus and as cells that produce and release mucus and other fluids, respectively. Despite recent medical advances, EC associated with distant metastasis and local invasion still leads to a poor prognosis. A recent statistical study revealed that the numbers of new EC cases and EC-related deaths worldwide in 2008 were estimated to be 482,300 and 406,800 , respectively [2]. This high mortality rate is largely due to poor subjective symptoms and difficulty with early diagnosis. Thus, EC is frequently diagnosed at late stages, leading to the unsatisfactory prognosis of affected patients, even though various therapeutic options such as surgery, chemotherapy, and radiotherapy are available. To date, endoscopic and radiologic examinations have been applied to detect EC at an early stage. Although the sensitivity and reliability of these diagnostic techniques have been improved, they do not always provide a satisfactory result. Furthermore, noninvasive diagnostic methods such as blood and urine tests for EC detection have not been established yet. Therefore, there is an urgent need to develop novel biomarkers for early detection and prognostic classification [3].

MicroRNAs (miRNAs) are small, noncoding RNAs of about 18-25 nucleotides in length that negatively regulate protein translation and/or mRNA stability by binding to target mRNAs. Although many miRNAs are expressed ubiquitously, a few are known to exhibit cell type/organ-dependent and/or developmental stage-dependent expression patterns 
[4]. Furthermore, aberrant miRNA expression has been found in various diseases, including many types of cancer [2]. Functionally, miRNAs act as either oncogenes or tumor suppressors via gene regulation and are thus called "oncomirs" [5, 6]. In silico target prediction revealed that individual miRNAs potentially target multiple mRNAs, indicating their crucial roles physiologically and pathologically. Thus, efforts to identify the actual target mRNAs of miRNAs experimentally are necessary to understand how miRNAs are associated with the onset and progression of various diseases, including cancer, possibly leading to improvements in current diagnostic and prognostic indicators. To achieve this goal, we used microarray analysis, which is a powerful tool because it can analyze the expression of a number of mRNAs and miRNAs in multiple samples at once.

Here, we present the datasets from our microarray experiments to analyze comprehensively and comparably mRNA and miRNA expression in normal and tumorous human esophageal squamous cell lines.

\section{Methodology}

Cell culture and RNA isolation were as follows. The ESCCderived human cell lines used in this study were TE-1, TE5 , and TE-8, which are derived from well, poorly, and moderately differentiated tumors, respectively. These cell lines were purchased from RIKEN Cell Bank (Tsukuba, Japan) and cultured in RPMI-1640 medium supplemented with $10 \%$ fetal bovine serum. Het-1A, an SV40 large T antigen-harboring normal human esophageal squamous cell line, was purchased from American Type Culture Collection (Manassas, VA, USA) and cultured using the BEGM kit (Lonza, Basel, Switzerland) but without the addition of the GA1000 (gentamycin-amphotericin B mix) provided with the kit. Total RNA was isolated from these cells using ISOGEN reagent (Wako, Osaka, Japan) according to the manufacturer's protocol. RNA purity was evaluated by the RNA integrity number (RIN), a representative index to assess RNA quality determined using the Agilent 2100 bioanalyzer (Agilent, Santa Clara, CA, USA). We confirmed RINs of more than 9.9 for all RNA samples used in this study (data not shown).

Microarray study design for mRNA expression analysis was as follows. The RNA samples isolated from Het-1A and the ESCC cells were subjected to microarray-based expression analyses for mRNA using a SurePrint G3 Human Gene Expression $8 \times 60 \mathrm{~K}$ v2 Array (Agilent). Cy3-labeled complementary RNA (cRNA) was prepared from $100 \mathrm{ng}$ total RNA from each sample using the Low Input Quick Amp Labeling Kit, one color (Agilent). For each sample, $0.6 \mu \mathrm{g}$ cRNA was hybridized using the Gene Expression Hybridization Kit (Agilent). The hybridized microarrays were subsequently washed using Gene Expression Wash Buffers Pack (Agilent) and subjected to fluorescent signal detection using a SureScan Microarray Scanner G4900DA (Agilent). The labeling, hybridization, and washing procedures were performed according to the manufacturer's instructions.

The intensities of the detected signals were quantified using Agilent Feature Extraction to generate raw data, which were registered in the Gene Expression Omnibus (GEO) database as accession number GSE61587. The quantified data were then normalized using GeneSpring GX12 (Agilent) to enable comparison of data from different microarrays.

Microarray study design for miRNA expression analysis was as follows. The RNA samples isolated from the normal and tumorous esophageal squamous cells were subjected to microarray-based expression analyses for miRNA using the miRCURY LNA microRNA Array Kit 7th generationhuman, mouse, and rat (Exiqon, Vedbaek, Denmark). Total RNA (250 ng) was $3^{\prime}$-labeled with Hy3 using the miRCURY LNA microRNA Hi-Power Labeling Kit (Exiqon) according to the manufacturer's instructions. For this labeling reaction, a synthesized miRNA mimic supplied in the Spike-in microRNA Kit v2 (Exiqon) was added to the reaction mixture to assess the quality of the obtained microarray data. The hybridized microarrays were subsequently washed according to the manufacturer's instructions and subjected to fluorescent signal detection using a SureScan Microarray Scanner G4900DA (Agilent).

The intensities of the detected signals were quantified using Agilent Feature Extraction to generate raw data, which were registered as GEO accession number GSE61588. The quantified data were then normalized using ImaGene 9 and Nexus Expression 3.0 (BioDiscovery) to enable comparison of data from different microarrays.

\section{Dataset Description}

The dataset associated with this Dataset Paper consists of 2 items which are described as follows.

Dataset Item 1 (Table). Normalized signal intensities from individual probes on SurePrint G3 Human Gene Expression $8 \times 60 \mathrm{~K}$ v2 Arrays. The figures in the Signal Evaluation columns represent the significance of these signal intensities as follows: 2 , detected; 1 , difficult to judge; 0 , not detected.

Column 1: Feature Number

Column 2: Probe ID

Column 3: Gene Symbol

Column 4: GenBank Accession Number

Column 5: Description

Column 6: Normalized Signal Intensity for Het-1A

Column 7: Signal Evaluation for Het-1A

Column 8: Normalized Signal Intensity for TE-1

Column 9: Signal Evaluation for TE-1

Column 10: Normalized Signal Intensity for TE-5

Column 11: Signal Evaluation for TE-5

Column 12: Normalized Signal Intensity for TE-8

Column 13: Signal Evaluation for TE-8

Column 14: GO Term

Dataset Item 2 (Table). Normalized signal intensities from individual probes on miRCURY LNA microRNA Arrays.

Column 1: Probe Number 
Column 2: miRNA

Column 3: Normalized Signal Intensity for Het-1A

Column 4: Normalized Signal Intensity for TE-1

Column 5: Normalized Signal Intensity for TE-5

Column 6: Normalized Signal Intensity for TE-8

\section{Concluding Remarks}

Many recent studies have revealed that miRNAs could be promising biomarkers as well as therapeutic targets for cancer. This is particularly important for cancer types with a poor prognosis, including ESCC. In the present study, we conducted microarray analysis to analyze miRNA expression comprehensively in a normal human esophageal squamous cell line and in three types of human ESCC-derived cell lines to identify candidate miRNAs aberrantly expressed in ESCC. Furthermore, we performed microarray-based comprehensive gene expression analysis in these cell lines. Although it is generally believed that miRNAs suppress the translation of their target mRNAs into proteins, recent studies have revealed that miRNA-mediated gene silencing largely involves mRNA degradation [7,8]. Thus, bioinformatic analyses of these microarray data, which include correlation analysis, miRNA target prediction, and ontology analysis, will provide an important clue to clarify how miRNAs are involved in the onset or progression of ESCC.

\section{Dataset Availability}

The dataset associated with this Dataset Paper is dedicated to the public domain using the CC0 waiver and is available at http://dx.doi.org/10.1155/2014/376541/dataset. In addition, the microarray data described herein (GSE61587 and GSE61588) were registered in GEO DataSets also as an integrated format as GSE61589. Deposited data are available at http://www.ncbi.nlm.nih.gov/geo/query/acc.cgi?acc= GSE61589.

\section{Conflict of Interests}

The authors declare that they have no conflict of interests.

\section{Acknowledgments}

This study was supported by Grant-in-Aid no. 24791449 from the Ministry of Education, Culture, Sports, Science and Technology (MEXT) and partly by the grant from Kurozumi Medical Foundation.

\section{References}

[1] P. C. Enzinger and R. J. Mayer, "Esophageal cancer," The New England Journal of Medicine, vol. 349, no. 23, pp. 2241-2252, 2003.

[2] A. Jemal, F. Bray, M. M. Center, J. Ferlay, E. Ward, and D. Forman, "Global cancer statistics," CA Cancer Journal for Clinicians, vol. 61, no. 2, pp. 69-90, 2011.
[3] M. B. Orringer, "Multimodality therapy for esophageal carcinoma-update," Chest, vol. 103, no. 4, pp. 406S-409S, 1993.

[4] D. P. Bartel, "MicroRNAs: genomics, biogenesis, mechanism, and function," Cell, vol. 116, no. 2, pp. 281-297, 2004.

[5] G. A. Calin and C. M. Croce, "MicroRNA signatures in human cancers," Nature Reviews Cancer, vol. 6, no. 11, pp. 857-866, 2006.

[6] R. Garzon, M. Fabbri, A. Cimmino, G. A. Calin, and C. M. Croce, "MicroRNA expression and function in cancer," Trends in Molecular Medicine, vol. 12, no. 12, pp. 580-587, 2006.

[7] H. Guo, N. T. Ingolia, J. S. Weissman, and D. P. Bartel, "Mammalian microRNAs predominantly act to decrease target mRNA levels," Nature, vol. 466, no. 7308, pp. 835-840, 2010.

[8] E. Huntzinger and E. Izaurralde, "Gene silencing by microRNAs: contributions of translational repression and mRNA decay," Nature Reviews Genetics, vol. 12, no. 2, pp. 99-110, 2011. 


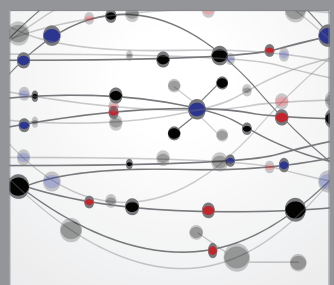

The Scientific World Journal
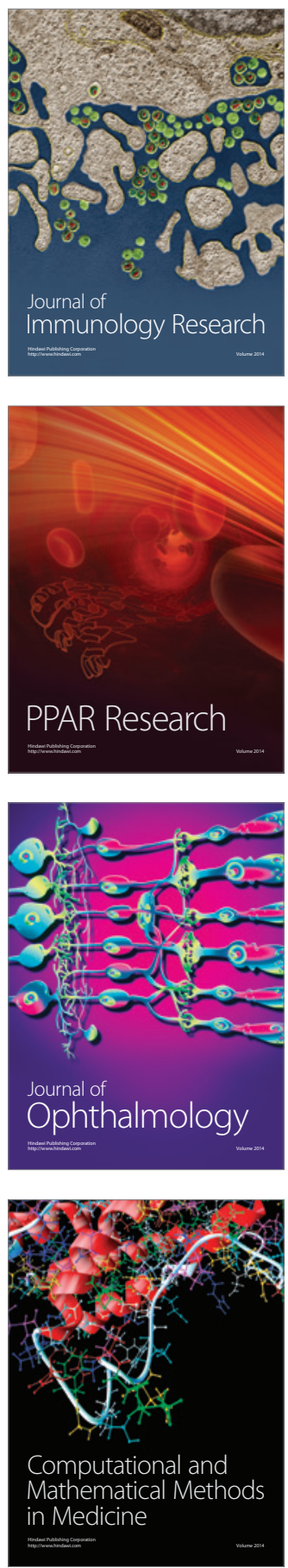

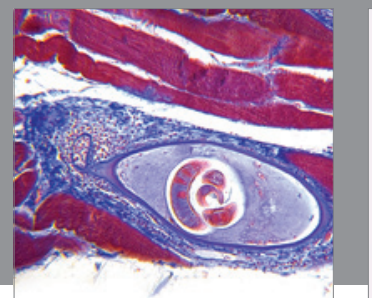

Gastroenterology

Research and Practice
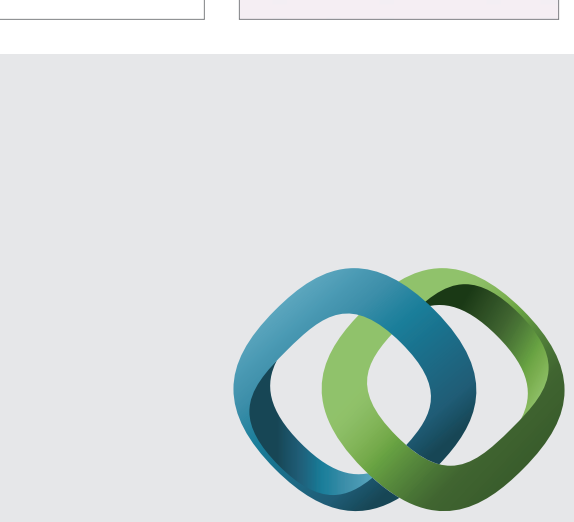

\section{Hindawi}

Submit your manuscripts at

http://www.hindawi.com
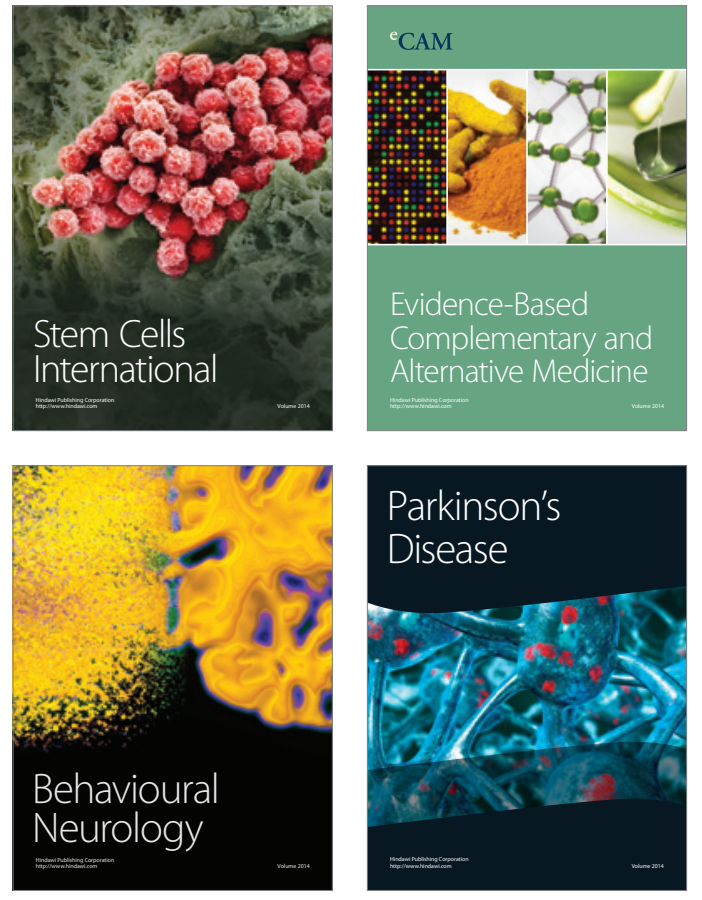
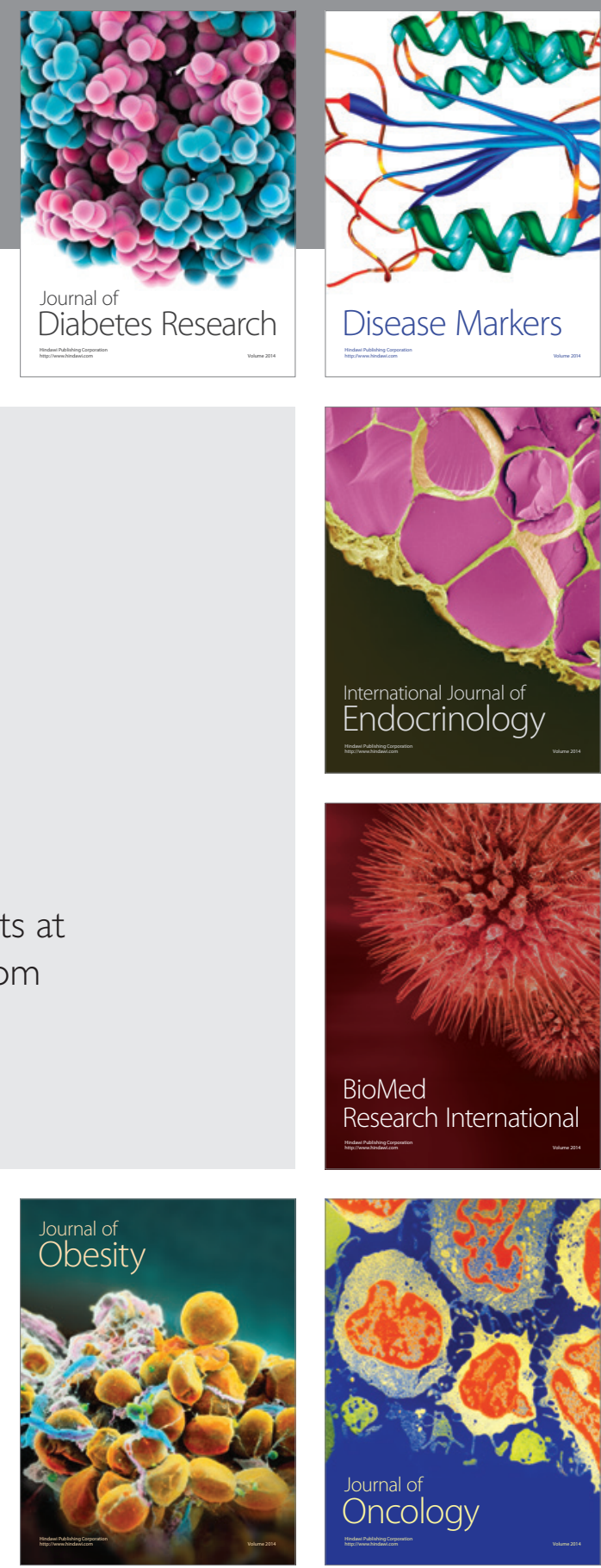

Disease Markers
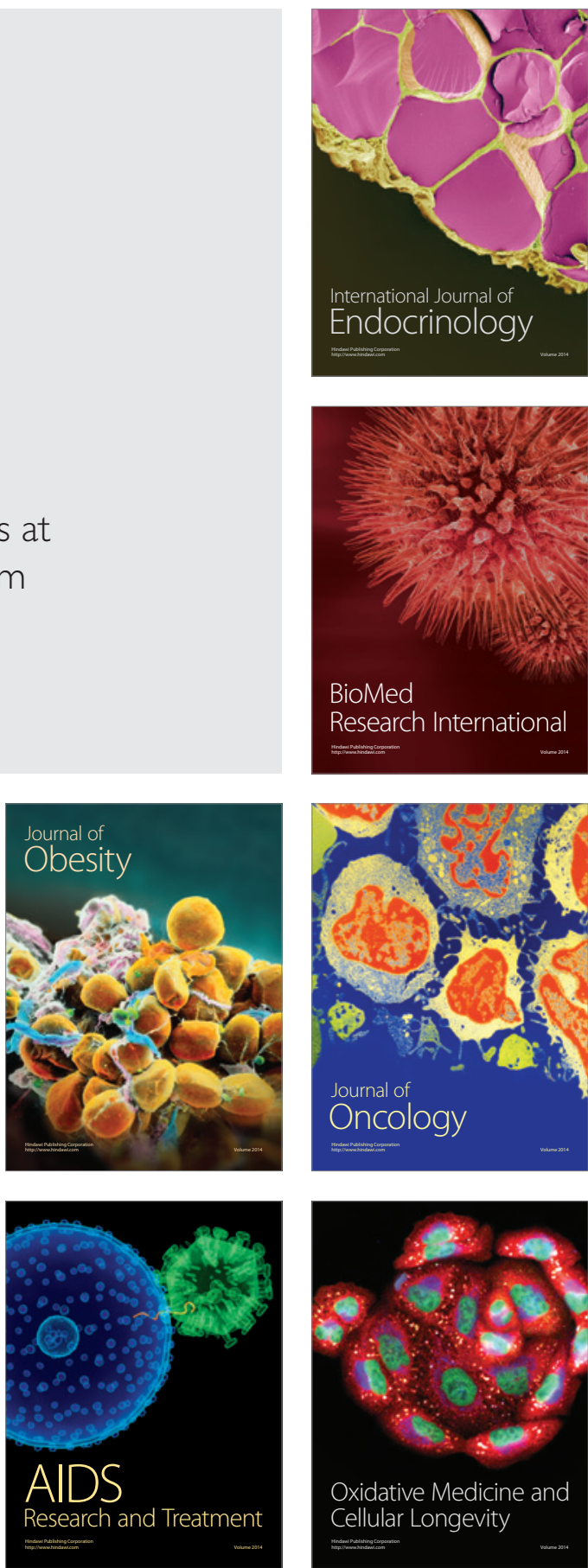